Kennesaw State University

DigitalCommons@Kennesaw State University

Faculty Publications

$8-1996$

\title{
New Promise for Electron Bulk Energization in Solar Flares: Preferential Fermi Acceleration of Electrons over Protons in Reconnection-driven Magnetohydrodynamic Turbulence
}

Ted La Rosa

Kennesaw State University, tlarosa1@kennesaw.edu

Ronald L. Moore

NASA

James A. Miller

University of Alabama - Huntsville

Steven N. Shore

Indiana University - South Bend

Follow this and additional works at: http://digitalcommons.kennesaw.edu/facpubs

Part of the Cosmology, Relativity, and Gravity Commons, Stars, Interstellar Medium and the Galaxy Commons, and the The Sun and the Solar System Commons

\section{Recommended Citation}

LaRosa, T.N., Moore, R.L., Miller, J.A., Shore, S.N. New promise for electron bulk energization in solar flares: preferential Fermi acceleration of electrons over protons in reconnection-driven magnetohydrodynamic turbulence. Astrophys. J. 467, 454-464, 1996.

This Article is brought to you for free and open access by DigitalCommons@Kennesaw State University. It has been accepted for inclusion in Faculty Publications by an authorized administrator of DigitalCommons@Kennesaw State University. For more information, please contact 
THE ASTROPHYSICAL JoURNAL, 467:454-464, 1996 August 10

(C) 1996. The American Astronomical Society. All rights reserved. Printed in U.S.A.

\title{
NEW PROMISE FOR ELECTRON BULK ENERGIZATION IN SOLAR FLARES: PREFERENTIAL FERMI ACCELERATION OF ELECTRONS OVER PROTONS IN RECONNECTION-DRIVEN MAGNETOHYDRODYNAMIC TURBULENCE
}

\author{
T. N. LARosA \\ Department of Biological and Physical Sciences, Kennesaw State College, Marietta, GA 30061 \\ R. L. MOORE \\ Solar Physics Branch/ES82, NASA/MSFC, Huntsville, AL 35812 \\ J. A. Miller \\ Department of Physics, University of Alabama in Huntsville, Huntsville, AL 35899 \\ AND \\ S. N. SHORE \\ Department of Physics and Astronomy, Indiana University at South Bend, South Bend, IN 46615 \\ Received 1995 August 25; accepted 1996 February 26
}

\begin{abstract}
The hard X-ray luminosity of impulsive solar flares indicates that electrons in the low corona are bulk energized to energies of order $25 \mathrm{keV}$. LaRosa \& Moore pointed out that the required bulk energization could be produced by cascading MHD turbulence generated by Alfvénic outflows from sites of strongly driven reconnection. LaRosa, Moore, \& Shore proposed that the compressive component of the cascading turbulence dissipates into the electrons via Fermi acceleration. However, for this to be a viable electron bulk energization mechanism, the rate of proton energization by the same turbulence cannot exceed the electron energization rate. In this paper we estimate the relative efficiency of electron and proton Fermi acceleration in the compressive MHD turbulence expected in the reconnection outflows in impulsive solar flares. We find that the protons pose no threat to the electron energization. Particles extract energy from the MHD turbulence by mirroring on magnetic compressions moving along the magnetic field at the Alfvén speed. The mirroring rate, and hence the energization rate, is a sensitive function of the particle velocity distribution. In particular, there is a lower speed limit $V_{\min } \approx V_{\mathrm{A}}$, below which the pitch-angle distribution of the particles is so highly collapsed to the magnetic field in the frame of the magnetic compressions that there is no mirroring and hence no Fermi acceleration. For coronal conditions, the proton thermal speed is much less than the Alfvén speed and proton Fermi acceleration is negligible. In contrast, nearly all of the electrons are super-Alfvénic, so their pitch-angle distribution is nearly isotropic in the frame of the magnetic compressions. Consequently, the electrons are so vigorously mirrored that they are Fermi accelerated to hard X-ray energies in a few tenths of a second by the magnetic compressions on scales of $10^{5}-10^{3} \mathrm{~cm}$ in the cascading MHD turbulence. We conclude that dissipation of reconnection-generated MHD turbulence by electron Fermi acceleration plausibly accounts for the electron bulk energization in solar flares.
\end{abstract}

Subject headings: acceleration of particles - MHD - Sun: flares - Sun: particle emission turbulence

\section{INTRODUCTION}

A solar flare is an explosive event in a magnetic region of the solar atmosphere. Apparently, stored magnetic energy is suddenly released low in the corona, driving bulk mass motion, heating plasma, and accelerating electrons and ions to hard X-ray energies and beyond (e.g., Sturrock 1980). In particular, the magnitude and energy spectrum of the resulting burst of hard X-rays often indicate that (1) much of the released energy goes to the energization of electrons to tens of kilovolts, (2) the number of these electrons in the burst is comparable to the total number of electrons in the preflare coronal volume of the flaring magnetic field, and (3) the total energy channeled into these electrons is a sizable fraction of the total preflare magnetic energy in the flare volume. The observed hard X-ray emission from flares thus confronts us with what is known as the electron bulk energization problem (Ramaty et al. 1980; Tandberg-Hanssen \& Emslie 1988; Melrose 1992; LaRosa \& Moore 1993): How does the flare energy-release process manage to be so highly efficient in energizing electrons to hard X-ray ener- gies? It is this difficulty that makes the plasma energization in solar flares one of the fundamental problems of plasma astrophysics (Rosner et al. 1991).

An enduring central idea in flare physics is that the conversion of magnetic energy into plasma particle energy is accomplished through reconnection of the magnetic field (e.g., Sweet 1969). Under this precept, the question becomes: How can reconnection provide the required electron bulk energization? LaRosa \& Moore (1993) pointed out that the main function of the reconnection could be to generate MHD turbulence that, in turn, bulk-energizes the electrons as it cascades to small scales. They concluded that the expected MHD turbulence is plausibly intense enough and voluminous enough to provide the electrons for the hard $\mathrm{X}$-ray emission. The question then becomes: How is the energy in this MHD turbulence dissipated into the electrons? In answer to this question, LaRosa, Moore, \& Shore (1994) proposed that the electrons are bulk energized by Fermi acceleration from the MHD turbulence at sufficiently small scales in the cascade. 
For the rate of Fermi-acceleration energization of the electrons by the turbulence, LaRosa et al. (1994) used the following equation from Melrose (1986, chap. 13):

$$
d E / d t=\pi \omega\left(V_{\mathrm{A}} / V_{\mathrm{rms}}\right)\left(\delta B / B_{0}\right)^{2} E,
$$

where $E$ is the mean energy of the particles, $V_{\mathrm{rms}}$ is the root mean square particle speed $\left(E=m V_{\mathrm{rms}}^{2} / 2\right), B_{0}$ is the ambient magnetic field strength, $V_{\mathrm{A}}$ is the Alfvén speed given by $B_{0}$ and the ambient mass density $\rho\left[V_{\mathrm{A}}=\right.$ $\left.B_{0} /(4 \pi \rho)^{1 / 2}\right], \delta B$ is the amplitude of the magnetic field compressions of length scale $\delta l$ that dominate the Fermi acceleration (these fluctuations propagate along the magnetic field at about the Alfvén speed $\left.V_{\mathrm{A}}\right)$, and $\omega$ is the frequency of these moving magnetic compressions $\left(\omega=V_{\mathrm{A}} / \delta l\right)$. By combining equation (1.1) with reasonable assumptions for the energy density and spectrum of the reconnection-driven MHD turbulence in flares, LaRosa et al. (1994) showed that the turbulence could bulk-energize the electrons to tens of kilovolts by dissipating at turbulence scales $\delta l$ on the order of a kilometer via Fermi acceleration of the electrons. This means that such turbulence-driven Fermi acceleration is a viable mechanism for the required electron bulk energization, provided that the turbulence cascades to kilometer scales before it dissipates.

In view of this requirement that the cascade not terminate at scales larger than those needed for the electron energization, equation (1.1) presents a possible threat from the protons. The plasma that comes into the flare reconnection region is expected to be thermal, so that the rms speed of the protons would be 40 times less than that of the electrons. Thus, if equation (1.1) applies to the protons as well as to the electrons, it appears that the turbulence would dissipate into the protons rather than into the electrons. In particular, if the MHD turbulence has a Kraichnan spectrum, $\delta B / B_{0}=\left(\delta l / l_{0}\right)^{1 / 4}$, then from equation (1.1) it appears that the turbulence would dissipate into the protons at scales of order $10^{3} \mathrm{~km}$, the protons thereby ending the cascade at scales far too long for appreciable energization of the electrons. The protons would be bulk-energized to tens of kilovolts, but the electrons would not. Thus, if equation (1.1) applied to protons as well as to the electrons, Fermiacceleration damping of the turbulence would fail to provide the electron energization required for the observed hard X-ray emission. (The estimated energy density of the turbulence is $25-50 \mathrm{keV}$ per particle. Electrons energized to 25-50 keV emit hard X-ray photons in this same energy range. However, protons must be energized to an energy $m_{p} / m_{e}$ times greater [or about $50 \mathrm{meV}$ ] to emit $25 \mathrm{keV}$ photons [Emslie \& Brown 1985]. Hence there is not sufficient energy density in the reconnection outflows to bulkenergize protons to hard X-ray-emitting energies and account for the observed X-ray emission). The viability of our turbulent cascade model for the electron bulk energization therefore hinges on the relative efficiency of electron and proton Fermi acceleration in our reconnection-driven MHD turbulence.

In LaRosa et al. (1994) we pointed out the potential problem of the protons absorbing the turbulent wave energy and blocking the electron energization. We also proposed an ad hoc solution to this problem. One of the requirements for Fermi acceleration is efficient pitch-angle scattering. The Fermi process boosts only the parallel momentum. Consequently the pitch-angle distribution compresses along the direction of the magnetic field.
Without pitch-angle scattering to repopulate the large pitch angles, the mirroring rate becomes negligible, there is no further wave-particle interaction, and the acceleration shuts off. We therefore suggested that in our reconnection-driven MHD turbulence the electrons could be strongly scattered by plasma right-circularly polarized waves (R-waves) (generated by the reconnection), while the protons experience negligible scattering and hence negligible Fermiacceleration energization. In this paper we show that Fermi-acceleration energization of the protons is negligible even if the protons are scattered as much as the electrons. This means that electron Fermi-acceleration dissipation of MHD turbulence is a more robust mechanism for electron bulk energization in solar flares than we previously thought.

In this paper we focus on the elements of Fermi acceleration by MHD turbulence. From these elements,-we heuristically derive the particle energization rate for the case in which the mean particle speed is much greater than the Alfvén speed, which is the case for the electrons in the flare turbulence. The rate that we derive is essentially that given by Melrose (1986) (our eq. [1.1] above). This agreement verifies our heuristic method of derivation. We then use the same method to derive an upper bound on the energization rate of the protons in the flare turbulence. In contrast to the electrons, the protons have an initial thermal speed that is much less than the Alfvén speed. Because of this, equation (1.1) does not apply to the protons. Instead, the proton energization rate is entirely negligible. Thus, we show that the protons pose no threat to the Fermi-acceleration bulk energization of the electrons by the MHD turbulence in solar flares.

In a much earlier work, Wentzel (1963) pointed out that electron Fermi acceleration by shocks could greatly exceed proton acceleration, since the proton thermal speed could be small compared to the shock speed. Since his work focused on shocks, not MHD waves, he did not explicitly calculate the particle energization rates. In this paper, we demonstrate the preferential Fermi acceleration of electrons over protons explicitly for Fermi acceleration by the compressive MHD waves in the cascading MHD turbulence expected in flares.

\section{PARTICLE ENERGIZATION BY FERMI ACCELERATION}

\subsection{Physical Picture of Fermi Acceleration}

The basic elements of Fermi acceleration are depicted in Figure 1. Through magnetic mirroring, particles exchange energy with compressive magnetic fluctuations propagating along the magnetic field with amplitude $\delta B$ and wavelength $\delta l$. However, it is only those particles with sufficiently large pitch angles, in the frame of the moving fluctuations, that mirror. It is assumed that the pitch-angle distribution in the plasma frame is isotropic. (The isotropy is presumably maintained by pitch-angle scattering caused by small-scale plasma turbulence, i.e., plasma waves with wavelengths of the order of the particle gyroradii; see $\S 4.2$. Thus, in the frame of a moving magnetic compression, the pitch-angle distribution is not isotropic. Instead, the particles en masse are seen by the moving compression as an oncoming beam; the greater the ratio of the magnetic compression wave speed to the average particle speed, the narrower the beam (see Fig. 2). There is a minimum pitch angle in the compression wave frame such that mirroring occurs only for particles with pitch angles exceeding this minimum. 


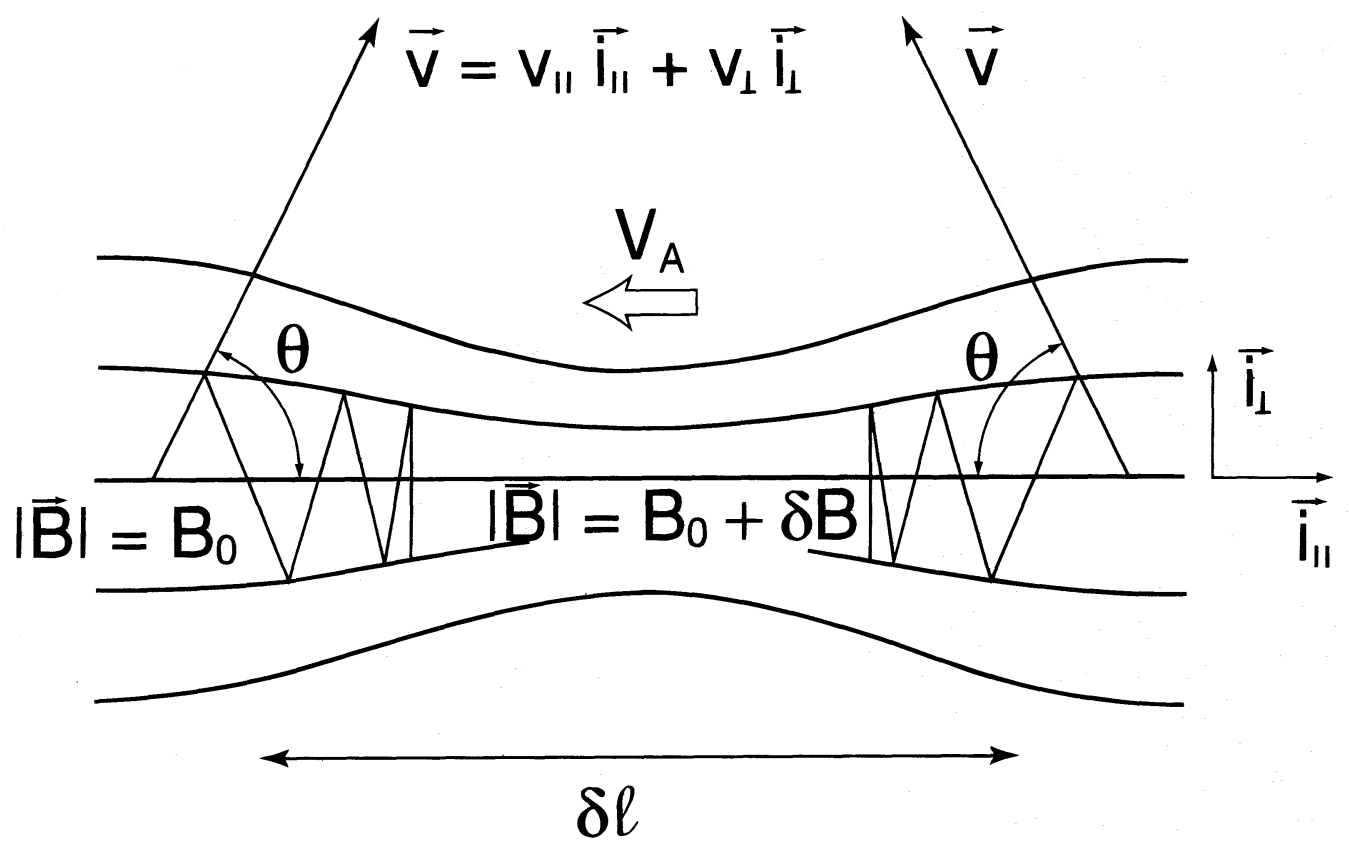

Fig. 1.-Depiction of the elementary wave-particle interactions involved in the Fermi-acceleration energization of particles by the compressive component of MHD turbulence. This depiction is in the plasma frame, the inertial frame of the MHD turbulence. In this specific example, a magnetic compression (the constriction in the bundle of magnetic field lines shown by the heavy lines) of length $\delta l$ and amplitude $\delta B$ propagates to the left along the ambient magnetic field $\boldsymbol{B}_{0}$ at the Alfvén speed $V_{\mathrm{A}}$. The unit vectors $\boldsymbol{i}_{\|}$and $\boldsymbol{i}_{\perp}$ are parallel and perpendicular to the ambient magnetic field. Also depicted are the velocity $\boldsymbol{V}$, pitch angle $\theta$, and trajectory up to the point of reflection for each of two reflecting particles: on the left, an oncoming particle meeting the compression; and on the right, an overtaking particle catching the compression from behind. The oncoming particle gains energy as it reflects from the compression, whereas the overtaking particle loses energy as it reflects.

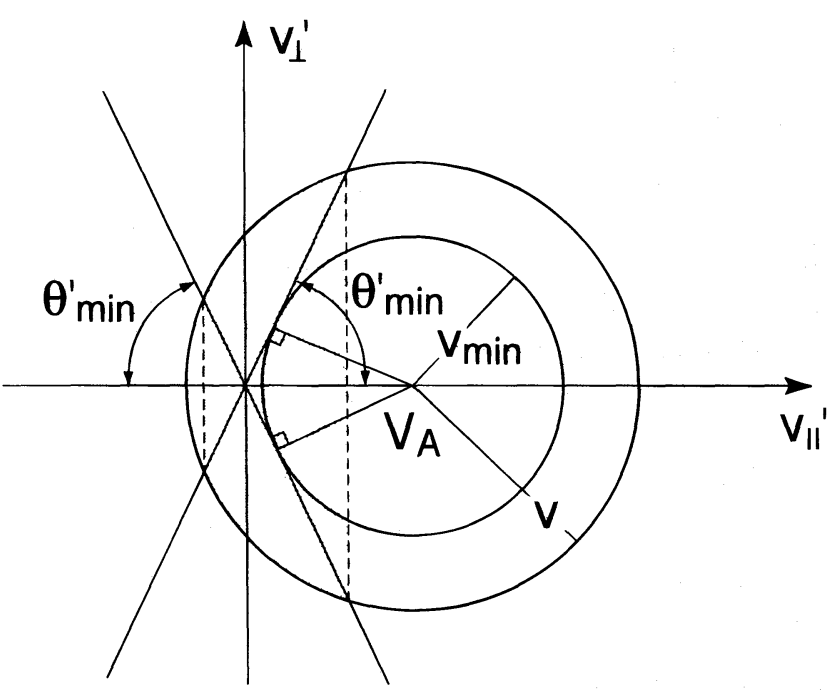

Fig. 2.-Particle-velocity diagram in the frame of a leftward-moving magnetic compression like that in Fig. 1. The compression ratio $\eta=$ $\left(B_{0}+\delta B\right) / B_{0}$ of the magnetic compression determines the minimum pitch angle $\theta_{\text {min }}^{\prime}$ (in the compression frame) that a particle can have and still be reflected: $\sin \theta_{\min }^{\prime}=\eta^{-1 / 2}$. The particles are spherically distributed about zero velocity in the plasma frame, so particles of any given speed $V$ are uniformly distributed on a sphere of radius $V$ centered on $\left(V_{\mathrm{A}}, 0\right)$ in the frame of the compression, as in this diagram. Particles on those parts of this sphere that are inside the two cones of half-angle $\theta_{\min }^{\prime}$ have pitch angles $\theta^{\prime}$ less than $\theta_{\min }^{\prime}$ and cannot be reflected by the magnetic compression. Particles on the rest of this sphere, the shaded zone in the diagram, are outside the two $\theta_{\min }^{\prime}$ cones, do have pitch angles $\theta^{\prime}$ greater than $\theta_{\min }^{\prime}$, and are reflected. Particles with $V_{\|}^{\prime}>0$ are oncoming particles; particles with $V_{\|}^{\prime}<0$ are overtaking particles. The diagram shows that any particle with speed less than $V_{\min }=V_{\mathrm{A}} \sin \theta_{\min }^{\prime}=V_{\mathrm{A}} / \eta^{1 / 2}$ cannot be reflected. It is also seen that particles with speeds $V \ll V_{\mathrm{A}}$ form a narrow oncoming beam in the frame of the moving magnetic compression and obviously cannot mirror.
Consequently, only a certain fraction of the particles will be reflected by a compression wave. The remaining particles (those with pitch angles smaller than the minimum for reflection) simply pass through the compression wave without any exchange of energy with it. For those particles that do have a large enough pitch angle to reflect, each individual reflection is either an oncoming (energy-gaining) or an overtaking (energy-losing) "collision." If the average particle speed is much greater than the wave speed, the rates on oncoming and overtaking collisions are practically equal. In this case, because the magnetic compressions are generated randomly in the MHD turbulence, and each compression carries much more energy than that of a reflecting particle, the particles experience a net gain in energy from the compression waves through random walk in energy. If the average particle speed is less than the wave speed, then there are few overtaking collisions in comparison to oncoming collisions, and instead of a random walk in energy, the reflecting particles gain energy in most collisions. However, the fraction of particles that interact in this case is much smaller, so the energization time greatly exceeds that in the random walk case. Thus, as we will show, the energization rate is a sensitive function of the particle velocity and pitch-angle distributions. We will heuristically derive the energization rates for two different particle speed regimes: when the mean particle speed greatly exceeds the wave speed (the case of the electrons in the flare turbulence) and when the mean particle speed is of the order of the wave speed or less (the case of the protons in the flare turbulence).

\subsection{Derivation of Particle Energization Rates}

Let $\tau_{E}$ denote the $e$-folding energization time for all of the 
particles (electrons or protons):

$$
\frac{1}{\tau_{E}}=\frac{1}{E} \frac{d E}{d t},
$$

where $E$ is the mean energy of the particles. Let $\tau_{E_{r}}$ denote the $e$-folding energization time of the reflected particles, i.e., $1 / \tau_{E_{r}}=1 / E_{r} d E_{r} / d t$, where $E_{r}$ is the average energy of the reflecting particles. If the particles are pitch-angle-scattered in less time than $\tau_{E_{r}}$, then

$$
\tau_{E}=\left(\tau_{E_{r}} / F\right) E / E_{r}
$$

where $F$ is the fraction of particles, both oncoming and overtaking, that mirror upon encountering a wave. We define $\delta t$ to be the average time between magnetic "collisions" of a reflecting particle and define $N$ to be the number of reflections per reflecting particle during a time $\tau_{E_{r}}$, so that $\tau_{E_{r}}=N \delta t$. Equation (2.2) can now be rewritten as

$$
\tau_{E}=\frac{N \delta t}{F}\left(\frac{E}{E_{r}}\right) .
$$

The parameters $N, \delta t, F$, and $E / E_{r}$ are all functions of the speed regime. For each of our two speed regimes $\left(V_{\mathrm{rms}} \gg V_{\mathrm{A}}\right.$ and $V_{\text {th }} \lesssim V_{\mathrm{A}}$ ), we can express these parameters in terms of $\delta l, \delta B / B_{0}, V_{\text {rms }}$, and $V_{\mathrm{A}}$.

$$
\text { 2.2.1. The Speed Regime } V_{\mathrm{rms}} \gg V_{\mathrm{A}}
$$

First consider the parameter $F$, the fraction of particles that mirror upon encountering a compression wave. Most of the compressive MHD fluctuations propagate along the magnetic field at about the Alfvén speed $V_{\mathrm{A}}$. Hence, the perpendicular particle velocities are unchanged in the compression frame and (to sufficient accuracy for our purpose) the parallel particle velocities transform to $V_{\|}^{\prime}=V_{\|}-V_{\mathrm{A}}$ for a rightward-moving compression and $V_{\|}^{\prime}=V_{\|}+V_{\mathrm{A}}$ for a leftward-moving compression (as in Figs. 1 and 2). We assume that in the plasma frame the particles are distributed isotropically in velocity space, so they are spherically distributed about the origin $V=0$. So, in the plasma frame and in the compression frame, particles of speed $V$ are uniformly distributed on the surface of a sphere of radius $V$. In the frame of a leftward-moving compression (Fig. 2) this sphere is centered on the point $V_{\|}^{\prime}=V_{\mathrm{A}}, V_{\perp}^{\prime}=0$. Particles with $V_{\|}^{\prime}>0$ are oncoming particles, and particles with $V_{\|}^{\prime}<0$ are overtaking the leftward-moving compression. In the frame of the moving compression, we denote the pitch angles of the oncoming particles by $\theta_{+}^{\prime}$ and the pitch angles of the overtaking particles by $\theta_{-}^{\prime}$. Each pitch angle $\theta_{+}^{\prime}$ defines a cone in the velocity space of the oncoming particles, and $\theta_{-}^{\prime}$ defines a cone in the velocity space of the overtaking particles. There is a minimum pitch angle for reflection: only those particles having pitch angles $\theta_{+}^{\prime}>$ $\theta_{\text {min }}^{\prime}$ are reflected by the moving compressions. Of the particles of speed $V$, only those in the speed-sphere zone defined by $\theta_{ \pm}^{\prime}>\theta_{\min }^{\prime}$ are reflected. The angles $\theta_{+}^{\prime}=\theta_{\min }^{\prime}$ and $\theta_{-}^{\prime}=\theta_{\min }^{\prime}$ determine the fraction of particles that are reflected at each speed $V$. This fraction $f\left(V, V_{\mathrm{A}}, \theta_{\min }^{\prime}\right)$ is the fraction of the area of the speed sphere of radius $V$ that is outside both of the two cones defined by $\theta_{+}^{\prime}=\theta_{\min }^{\prime}$ and $\theta_{-}^{\prime}=\theta_{\min }^{\prime}$, the fraction of the speed-sphere area covered by the zone defined by $\theta_{ \pm}^{\prime}>\theta_{\min }^{\prime}$ (see Fig. 2). Consequently at particle speeds below a certain minimum $V_{\min }$, no particles are reflected. At $V=V_{\min }$, the speed sphere is tangent to the velocity cone defined by $\theta_{+}^{\prime}=\theta_{\min }^{\prime}$.
The minimum pitch angle for reflection $\theta_{\min }^{\prime}$ is a consequence of the conservation of the magnetic moment of a spiraling particle and is determined entirely by the compression ratio of the magnetic fluctuation (e.g., Spitzer 1962),

$$
\sin ^{2} \theta_{\min }^{\prime}=1 / \eta,
$$

where $\eta=\left(B_{0}+\delta B\right) / B_{0}$ is the magnetic compression ratio. As can be seen from Figure $2, V_{\min }=V_{\mathrm{A}} \sin \theta_{\min }^{\prime}$; therefore, $V_{\min }=V_{\mathrm{A}} / \eta^{1 / 2}$. At speeds $V<V_{\min }$, all particles are oncoming particles and none are reflected. At speeds $V_{\min }<V<V_{\mathrm{A}}$ all particles are oncoming particles, and some are reflected. At speeds $V>V_{\mathrm{A}}$ there are both oncoming and overtaking particles, and some of each are reflected. Clearly, it is the ratio of the average particle speed to the Alfvén speed that largely determines what fraction of the particles will mirror. For average particle speeds much below the Alfvén speed the pitch-angle distribution in the wave frame is so compressed along the magnetic field direction that mirroring is negligible.

Denote by $F_{+}$the fraction of oncoming particles that reflect (those having $\theta_{+}^{\prime}>\theta_{\text {min }}^{\prime}$ ), and denote by $F_{-}$the fraction of overtaking particles that reflect (having $\theta_{-}^{\prime}>\theta_{\min }^{\prime}$ ), so that the total fraction that reflect is $F=F_{+}+F_{-}$. For the present case of $V_{\mathrm{rms}} \gg V_{\mathrm{A}}$, it is seen from the velocity diagram (Fig. 2) that $F_{+} \approx F_{-}$, hence $F \approx 2 F_{+}$.

$$
F_{+} \approx a_{z} / a_{s},
$$

where $a_{z}$ is the area of the zone of the $V_{\text {rms }}$ sphere occupied by oncoming reflecting electrons and $a_{s}$ is the area of the whole $V_{\mathrm{rms}}$ sphere. The area of the zone is $2 \pi R h$, where $R=V_{\mathrm{rms}}$ and (from Fig. 2) for $V_{\mathrm{rms}} \gg V_{\mathrm{A}}, h \approx V_{\mathrm{rms}} \cos \theta_{\mathrm{min}}^{\prime}$. Dividing the area of the zone by the area of the whole sphere $4 \pi R^{2}, \quad F_{+} \approx \frac{1}{2} \cos \theta_{\min }^{\prime}$ and $F \approx \cos \theta_{\min }^{\prime}$. For $\delta B / B_{0} \ll 1, \quad \cos \theta_{\min }^{\prime} \approx\left(\delta B / B_{0}\right)^{1 / 2}$, and we obtain $F \approx$ $\left(\delta B / B_{0}\right)^{1 / 2}$.

Next consider $E / E_{r}$. For $V_{\mathrm{rms}} \gg V_{\mathrm{A}}$, the average energy of the reflecting particles is practically the same as the average energy of all the particles (see Fig. 2): $E_{r} \approx E$, so $\tau_{E} \approx$ $N \delta t / F$.

Now turn to $\delta t$, the time required for an average reflecting particle to traverse one wavelength $\delta l$. Denote by $\left\langle\left|V_{\|}^{\prime}\right|\right\rangle_{\theta_{土^{\prime}}>\theta_{\min ^{\prime}}}$ the average speed along the magnetic field of reflecting particles in the compression frame. In this

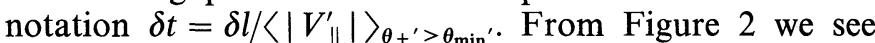
that for $V_{\mathrm{rms}} \gg V_{\mathrm{A}},\left\langle\left|V_{\|}^{\prime}\right|\right\rangle_{\theta_{土^{\prime}}>\theta_{\min ^{\prime}}} \approx \frac{1}{2} V_{\mathrm{rms}} \cos \theta_{\min }^{\prime}$. For weak magnetic compressions $\cos \theta_{\min }^{\prime} \approx\left(\delta B / B_{0}\right)^{1 / 2}$ and $\delta t \approx 2 \delta l /\left[V_{\mathrm{rms}}\left(\delta B / B_{0}\right)^{1 / 2}\right]$.

Finally, we turn to expressing $N$. In the case of $V_{\mathrm{rms}} \gg V_{\mathrm{A}}$ the average energy gained in an oncoming collision practically equals the energy lost in an overtaking collision, $\left\langle\delta E_{+}\right\rangle \approx\left\langle-\delta E_{-}\right\rangle$. This means that the particles gain energy from the turbulent fluctuations by random walk. For a random walk the number of reflections $N$ for the average reflecting electron to gain an $e$-fold increase in its energy is given approximately by

$$
N \approx\left(E /\left\langle\left|\delta E_{ \pm}\right|\right\rangle\right)^{2}
$$

From energy and momentum conservation in the average oncoming collision and in the average overtaking collision, we obtain $\left\langle\left|\delta E_{ \pm}\right|\right\rangle \approx 2 m V_{\mathrm{A}}\left\langle\left|V_{\|}^{\prime}\right|\right\rangle_{\theta_{ \pm^{\prime}}>\theta_{\min ^{\prime}} \text {. As indicated }}$ above, for $V_{\text {rms }} \gg V_{\mathrm{A}}$ and $\delta B / B_{0} \ll 1,\left\langle\left|V_{\|}^{\prime}\right|\right\rangle_{\theta_{土^{\prime}}>\theta_{\min ^{\prime}} \approx} \approx$ $\frac{1}{2} V_{\mathrm{rms}} \cos \theta_{\min }^{\prime} \approx \frac{1}{2} V_{\mathrm{rms}}\left(\delta B / B_{0}\right)^{1 / 2}$, so that $\left\langle\left|\delta E_{ \pm}\right| \approx\right.$ $m V_{\mathrm{A}} V_{\mathrm{rms}}\left(\delta B / B_{0}\right)^{1 / 2}$. Using $E=m V_{\mathrm{rms}}^{2} / 2$, we then obtain $N \approx \frac{1}{4}\left(V_{\mathrm{rms}} / V_{\mathrm{A}}\right)^{2} B_{0} / \delta B$. 
Substitution of our expressions for $N, F$, and $\delta t$ into equation (2.3) yields

$$
\tau_{E} \approx \frac{1}{2}\left(\frac{\delta l}{V_{\mathrm{A}}}\right)\left(\frac{V_{\mathrm{rms}}}{V_{\mathrm{A}}}\right)\left(\frac{B_{0}}{\delta B}\right)^{2} .
$$

Recall that $\tau_{\hat{E}}=[(1 / E) d E / d t]^{-1}$ and $V_{\mathrm{A}} / \delta l \approx \omega$, the frequency of the magnetic compressions. Using these substitutions, we can express the energization rate as

$$
d E / d t \approx 2 \omega\left(V_{\mathrm{A}} / V_{\mathrm{rms}}\right)\left(\delta B / B_{0}\right)^{2} E .
$$

Because this expression agrees with the standard expression given by Melrose (1986) (our eq. [1.1]) to within the small constant factor $\pi / 2$, we are confident that our heuristic method of derivation is valid. We stress that this expression applies only in the regime $V_{\text {rms }} \gg V_{\mathrm{A}}$. However, in the locale of flare reconnection in active regions this condition is well satisfied by the electrons.

$$
\text { 2.2.2. The Speed Regime } V_{\mathrm{th}} \lesssim V_{\mathrm{A}}
$$

Equation (2.7) for the $e$-folding energization time $\tau_{E}$ applies to the electrons in our flare MHD turbulence throughout their Fermi-acceleration energization from their initial energy $E_{0}(\sim k T \sim 0.3 \mathrm{keV})$ to their final mean energy $E_{f}(\sim 20 \mathrm{keV})$. Using $E=m_{e} V_{\mathrm{rms}}^{2} / 2$, we can integrate equation (2.8) to find the acceleration time for the electrons to reach $E_{f}$. This time, together with the requirement that this time match the cascade time (approximately equal to the large-eddy turnover time) of the MHD turbulence, sets the dissipation scale at which the turbulent cascade terminates by Fermi acceleration of the electrons. To show that the protons gain practically no energy as the compressive component of the turbulence dissipates on the electrons, it is sufficient to show that the initial $e$-folding energization time $\tau_{E_{0}}$ for the electrons is much shorter than the initial $e$-folding time for the Fermi-acceleration energization of the protons in the same MHD turbulence. (Both the electrons show that there is negligible Fermi acceleration of the protons, we now turn to deriving a lower bound on $\tau_{E_{0}}$ for the particle speed regime that applies to the protons in the flare MHD turbulence, namely, $V_{\mathrm{th}} \lesssim V_{\mathrm{A}}$.

It is convenient to first consider the more limited speed regime $V_{\mathrm{th}} \lesssim V_{\mathrm{A}}-V_{\min }$. In this regime we will estimate a lower bound on $\tau_{E_{0}}$ expressed in the same terms as $\tau_{E}$ in equation (2.7) $\left(\delta l, \delta B / B_{0}, V_{\mathrm{th}}, V_{\mathrm{A}}\right)$ and under the assumption of weak magnetic compressions (small $\delta B / B_{0}$ and large $\theta_{\min }^{\prime}$ ). We will then modify this expression so that it gives a lower bound on $\tau_{E_{0}}$ throughout the full speed regime $V_{\mathrm{th}} \lesssim$ $V_{\mathrm{A}}$. This lower bound on $\tau_{E_{0}}$ for the protons will then be compared with the $\tau_{E_{0}}$ given by equation (2.7) for the electrons.

Recall $\tau_{E}=\left(E / E_{r}\right) N \delta t / F$. We determine first an expression for $E / E_{r}$, where $E=m V_{\mathrm{rms}}^{2} / 2$, and $E_{r}$ is the mean kinetic energy of the reflected particles. Let the ambient particles in the MHD turbulence initially have a thermal speed distribution

$$
\Lambda(V, T)=\left(4 / \pi^{1 / 2}\right)\left(V / V_{\mathrm{th}}\right)^{2} \exp \left[-\left(V / V_{\mathrm{th}}\right)^{2}\right] / V_{\mathrm{th}},
$$

where $m V_{\mathrm{th}}^{2} / 2=k T$. Initially $V_{\mathrm{rms}} \approx V_{\mathrm{th}}$, and $E \approx m V_{\mathrm{th}}^{2} / 2$. From $V_{\min }=V_{\mathrm{A}} / \eta^{1 / 2}$ and $\eta=1+\delta B / B_{0}$, for weak magnetic compressions $V_{\mathrm{A}}-V_{\min } \approx \frac{1}{2}\left(\delta B / B_{0}\right) V_{\mathrm{A}} \ll V_{\mathrm{A}}$. From the velocity diagram (Fig. 2) it is seen that for $V_{\text {th }} \ll V_{\mathrm{A}}, E_{r} \approx$ $m V_{\mathrm{A}}^{2} / 2$, so that $E / E_{r} \approx\left(V_{\mathrm{th}} / V_{\mathrm{A}}\right)^{2}$.

We next determine $\delta t$ from $\delta t=\delta l /\left\langle\left|V_{\|}^{\prime}\right|\right\rangle_{\theta_{t^{\prime}}>\theta_{\min ^{\prime}}}$. For $V_{\text {th }} \ll V_{\mathrm{A}}$ it can be inferred from the velocity diagram (Fig. 2) that there are many more reflecting oncoming particles than there are reflecting overtaking particles. Hence,

$$
\left\langle\left|V_{\|}^{\prime}\right|\right\rangle_{\theta_{ \pm^{\prime}}>\theta_{\min ^{\prime}}} \approx\left\langle\left|V_{\|}^{\prime}\right|\right\rangle_{\left.\boldsymbol{\theta}_{+^{\prime}}\right\rangle \boldsymbol{\theta}_{\min ^{\prime}}} .
$$

For large $\theta_{\min }^{\prime}$ and $V_{\mathrm{th}}<V_{\mathrm{A}}-V_{\min }$, it can be seen from the velocity diagram that

$$
\left\langle\left|V_{\|\|}^{\prime}\right|\right\rangle_{\theta_{+^{\prime}}>\theta_{\min }{ }^{\prime}} \approx V_{\mathrm{A}}-V_{\min } \text {. }
$$

Using $V_{\mathrm{A}}-V_{\min } \approx \frac{1}{2}\left(\delta B / B_{0}\right) V_{\mathrm{A}}$, we obtain

$$
\delta t \approx 2 \delta l /\left(V_{\mathrm{A}} \delta B / B_{0}\right) \text {. }
$$

Our next task is to obtain an expression for $F$, the fraction of particles that mirror upon encountering a wave. Note that $F=F_{+}+F_{-}$, where $F_{+}$represents the oncoming particles and $F_{-}$the overtaking particles. For $V_{\text {th }} \lesssim V_{\mathrm{A}}-V_{\min }$, the velocity diagram indicates that $F_{+} \gg$ $F_{-}$, so that $F \approx F_{+}$. Let $f_{+}\left(V, V_{\mathrm{A}}, \eta\right)=$ the fraction of oncoming particles at speed $V$ that are reflected by magnetic compressions of compression ratio $\eta$. In terms of $f_{+}\left(V, V_{\mathrm{A}}, \eta\right)$

$$
F_{+}=\int_{V_{\min }}^{\infty} f_{+}\left(V, V_{\mathrm{A}}, \eta\right) \Lambda(V, T) d V .
$$

It an be shown from the velocity diagram that the maximum of $f_{+}\left(V, V_{\mathbf{A}}, \eta\right)$ is approximately $\frac{1}{2} \cos \theta_{\min }^{\prime}$. Therefore, for weak magnetic compressions we have

$$
F<\frac{1}{2}\left(\frac{\delta B}{B_{0}}\right)^{1 / 2} \int_{V_{\min }}^{\infty} \Lambda(V, T) d V
$$

Using this approximation

$$
\int_{V_{\min }}^{\infty} \Lambda(V, T) d V \approx \frac{V_{\mathrm{A}}}{V_{\mathrm{th}}} \exp \left[-\left(\frac{V_{\mathrm{A}}}{V_{\mathrm{th}}}\right)^{2}\right],
$$

we obtain

$$
F \lesssim \frac{1}{2}\left(\frac{\delta B}{B_{0}}\right)^{1 / 2}\left(\frac{V_{\mathrm{A}}}{V_{\mathrm{th}}}\right) \exp \left[-\left(\frac{V_{\mathrm{A}}}{V_{\mathrm{th}}}\right)^{2}\right] .
$$

The final parameter required for our lower bound on $\tau_{E_{0}}$ is $N$, the number of reflections for the average reflecting particle to gain an $e$-fold increase in energy. In the speed regime $V_{\mathrm{th}}<V_{\mathrm{A}}-V_{\min }$, the oncoming reflecting particles greatly outnumber the overtaking reflecting particles. Hence the number of reflections for the average reflecting electron to gain an $e$-fold increase in energy is given approximately by

$$
N \approx E_{r} /\left\langle\delta E_{+}\right\rangle \text {. }
$$

For $\quad V_{\text {th }} \lesssim V_{\mathrm{A}}-V_{\min }, \quad E_{r} \approx m V_{\mathrm{A}}^{2} / 2$ and $\left\langle\delta E_{+}\right\rangle \approx$ $m\left(\delta B / B_{0}\right) V_{A}^{2}$, so that $N \approx B_{0} / 2 \delta B$. Substituting in equation (2.3) for $\tau_{E}$ our above approximate expressions for $E / E_{r}, \delta t$, and $N$, and our upper bound on $F$, we obtain the following lower bound on $\tau_{E_{0}}$ :

$$
\tau_{E_{0}} \gtrsim 2\left(\delta l / V_{\mathrm{A}}\right)\left(V_{\mathrm{th}} / V_{\mathrm{A}}\right)^{3}\left(B_{0} / \delta B\right)^{5 / 2} \exp \left(V_{\mathrm{A}} / V_{\mathrm{th}}\right)^{2}
$$

for the particle speed regime $V_{\text {th }} \lesssim V_{\mathrm{A}}-V_{\text {min }}$.

Finally, we can use the velocity diagram (Fig. 2) together with the thermal speed distribution (eq. [2.9]) in the same manner that was used to derive the above lower bound on $\tau_{E_{0}}$, to derive, from $\tau_{E}=\left(E / E_{r}\right) N \delta t / F$, a lower bound on $\tau_{E_{0}}$ that is valid over the extended speed regime $V_{\text {th }} \lesssim V_{\mathrm{A}}$. We 
use the same approximation for the ratio of the mean energy of all the particles to the mean energy of the reflecting particles: $E / E_{r} \approx\left(V_{\mathrm{th}} / V_{\mathrm{A}}\right)^{2}$. Instead of equation (2.12) for $\delta t$, for the extended speed range we obtain the following lower bound on $\delta t$ :

$$
\delta t \gtrsim\left(\delta l / V_{\mathrm{A}}\right)\left(\delta B / B_{0}\right)^{-1 / 2} .
$$

In place of $F \approx F_{+}$, we instead have for the extended speed range $F<2 F_{+}$, from which we obtain $F \lesssim$ $\left(\delta B / B_{0}\right)^{1 / 2}\left(V_{\mathrm{A}} / V_{\mathrm{th}}\right) \exp \left[-\left(V_{\mathrm{A}} / V_{\mathrm{th}}\right)^{2}\right]$. Finally, for $V_{\mathrm{th}} \sim V_{\mathrm{A}} \underset{\mathrm{it}}{\overparen{1}}$ can be seen from the velocity diagram that the number of overtaking reflecting particles is less than the number of reflecting oncoming particles but of the same order. Hence, for the extended speed range, $V_{\mathrm{th}} \lesssim V_{\mathrm{A}}$, the number of reflections for the average reflecting particle to gain and $e$-fold increase in energy lies between the limits given by the case of $V_{\mathrm{th}} \gg V_{\mathrm{A}}$ and the case $V_{\mathrm{th}} \lesssim V_{\mathrm{A}}-V_{\text {min }}$ :

$$
\left(E_{r} /\left\langle\delta E_{+}\right\rangle\right)^{2}>N \gtrsim E_{r} /\left\langle\delta E_{+}\right\rangle \text {. }
$$

So, for an upper bound on $\tau_{E_{0}}$, we use $N \gtrsim E_{r} /\left\langle\delta E_{+}\right\rangle$. In the extended speed range, we again have $E_{r} \sim m V_{A}^{2} / 2$, but in place of $\left\langle\delta E_{+}\right\rangle \approx m V_{A}^{2}\left(\delta B / B_{0}\right)$ (valid for $\left.V_{\mathrm{th}} \lesssim V_{\mathrm{A}}-V_{\min }\right)$, we now have $\left\langle\delta E_{+}\right\rangle \lesssim 2 m V_{\mathrm{A}}^{2}\left(\delta B / B_{0}\right)^{1 / 2}$, which gives $N \gtrsim$ $\frac{1}{4}\left(\delta B / B_{0}\right)^{-1 / 2}$. With these bounds on $\delta t, F$ and $N$, our lower bound on the initial energization time $\tau_{E_{0}}$ becomes

$$
\tau_{E_{0}} \gtrsim \frac{1}{4} \frac{\delta l}{V_{\mathrm{A}}}\left(\frac{V_{\mathrm{th}}}{V_{\mathrm{A}}}\right)^{3}\left(\frac{B_{0}}{\delta B}\right)^{3 / 2} \exp \left(\frac{V_{\mathrm{A}}}{V_{\mathrm{th}}}\right)^{2}
$$

for the extended speed regime $V_{\mathrm{th}}<V_{\mathrm{A}}$.

In our reconnection-driven MHD turbulence (in a flare in the low corona in an active region), for representative orderof-magnitude values of the ambient magnetic field strength and electron density, we take $B_{0} \sim 10^{2} \mathrm{G}$ and $n_{e} \sim 10^{10}$ $\mathrm{cm}^{-3}$ (e.g., Moore, LaRosa, \& Orwig 1995). These quantities set the Alfvén speed: $V_{\mathrm{A}} \sim 2 \times 10^{8} \mathrm{~cm} \mathrm{~s}^{-1}$. For an Alfvén speed of this order, the electrons entering the turbulence are in the speed regime $V_{\mathrm{th}} \gg V_{\mathrm{A}}$ if they have a temperature $T_{0} \lesssim 10^{6} \mathrm{~K}$, which is quite likely, and the protons are in the speed regime $V_{\mathrm{th}} \lesssim V_{\mathrm{A}}$ if they have a temperature $T_{0} \lesssim 10^{8} \mathrm{~K}$, which is certain. Thus, using equation (2.7) for $\tau_{E_{0}}$ for the electrons and equation (2.20) for a lower bound on $\tau_{E_{0}}$ for the protons, we can compare the initial energization time for the electrons with that for the protons for any initial plasma temperature in the range $10^{6} \lesssim T_{0} \lesssim$ $10^{8} \mathrm{~K}$.

\section{APPLICATION TO SOLAR FLARES}

We are now ready to evaluate the bulk energization of electrons by Fermi acceleration from the MHD turbulence expected in the impulsive phase of solar flares. We will find that the Fermi acceleration acts fast enough to be the damping mechanism for the compressive component of the turbulence - that is, that Fermi acceleration becomes fast enough at short enough scales in the turbulent cascade to end the cascade by dissipating the cascading turbulent energy into random-velocity kinetic energy of the electrons. We will also find that at this termination scale the Fermi acceleration acts much faster on the electrons than on the protons, so that practically all of the energy of the compressive turbulence is absorbed by the electrons while the protons get practically none. To bring out these results, we begin by specifying the magnitude and spectrum of the MHD turbulence that we expect to be generated by strong- ly driven reconnection in the impulsive phase of flares, and that is compatible with observed characteristics of impulsive-phase hard X-ray bursts. From this, we determine the dissipative end scale of the cascading-turbulence spectrum, at which scale the turbulence dissipates itself by Fermi accelerating the electrons. For this same turbulence with the same termination scale, we then show that the Fermi-acceleration energization rate of the protons is negligible compared to that of the electrons for any plausible temperature of the coronal plasma entering the turbulent cascade.

\subsection{Specification of the MHD Turbulence}

Coronal and chromospheric observations of the spatial development of flares suggest that in many large flares the magnetic energy release occurs through reconnection, and that this reconnection is strongly driven during the impulsive phase of the flare (e.g., Moore et al. 1984; Moore \& Roumeliotis 1992; Moore et al. 1995). In strongly driven reconnection, the outflows expelled by the reconnected field have speeds of the order of the Alfvén speed given by the strength of this field (e.g., Priest \& Forbes 1992). As in our previous work on electron bulk energization in flares (LaRosa \& Moore 1993; LaRosa et al. 1994; Moore et al. 1995), we assume that strongly driven reconnection is inherent in the impulsive-phase flare energy release, that the Alfvénic outflows are fully turbulent, that at each scale in this cascading turbulence the fluctuations or eddies have equipartition of kinetic and magnetic energy, and that the cascade produces a Kraichnan (1965) spectrum of fluctuations:

$$
\begin{gathered}
\delta V / V_{0}=\delta B / B_{0}=\left(\delta l / l_{0}\right)^{1 / 4} \\
{\left[\delta V / V_{0}=\delta B / B_{0}=\left(\delta l / l_{0}\right)^{1 / 3}\right],}
\end{gathered}
$$

where $V_{0}, B_{0}$, and $l_{0}$ are the velocity fluctuation amplitude, magnetic field fluctuation amplitude, and diameter of the largest eddies (those at the top of the cascade), and $\delta V$ and $\delta B$ are the fluctuation amplitudes of the eddies of diameter $\delta l$ within the cascade. Because the reconnection outflows are Alfvénic, $V_{0}$ is of the order of the Alfvén speed in the reconnecting field and $B_{0}$ is of the order of the strength of this field. (There is some evidence, from observations of the solar wind and from computer simulations, that the spectrum of MHD turbulence may be closer to a Kolmogorov spectrum than a Kraichnan spectrum [Verma 1994; Miller $\&$ Roberts 1996]. It is reasonable to assume that if the spectrum of our flare turbulence is not as flat as a Kraichnan spectrum, it is not steeper than a Kolmogorov spectrum. In this section, wherever the spectrum affects the outcome, following each result for a Kraichnan spectrum the result for a Kolmogorov spectrum is given in brackets, as in eq. [3.1] above.)

In the coronal body of active regions that flare, the field strength is $\gtrsim 10^{2} \mathrm{G}$ (Martres \& Bruzek 1977; Moore 1992; Moore et al. 1995), and the preflare coronal plasma has an electron density $n_{e} \sim 10^{10} \mathrm{~cm}^{-3}$ and temperature $T_{0} \lesssim$ $3 \times 10^{6} \mathrm{~K}$ (Moore \& Fung 1972; Vaiana \& Rosner 1978). Therefore, in the reconnecting magnetic fields in flares, we expect the plasma beta (ratio of gas pressure to magnetic pressure) to be small: $16 \pi n_{e} k T_{0} / B_{0}^{2} \lesssim 10^{-2}$. In MHD turbulence in low-beta plasma the fluctuations are largely comprised of two wave modes: (1) compressive or fast-mode waves and (2) noncompressive Alfvén waves (Melrose 1986). 
We assume that in the reconnection-driven low-beta turbulence in flares there is a rough equipartition between these two modes: about half of the energy of the turbulence resides in compressive fluctuations and about half in Alfvén waves. The Fermi acceleration directly dissipates only the compressive part of the turbulence. Even so, more than half of the total energy of the turbulence can be dissipated by Fermi acceleration: the half that is already in the compressive modes in the nondissipative range of the cascade (at scales larger than the Fermi dissipation scale), plus some part of the energy in the Alfvén modes, the part that couples into compressive modes as the Alfvén modes cascade beyond the Fermi-accelerated dissipation scale. In essence, the implication of equipartition is that of the order of the entire energy of the turbulence is available for dissipation by Fermi acceleration.

Assuming that the impulsive-phase reconnection outflows have speeds of the order of the Alfvén speed $V_{\mathrm{A}}=$ $B_{0} /(4 \pi \rho)^{1 / 2}$ and are fully turbulent, the energy density of this MHD turbulence is of order $\rho V_{\mathrm{A}}^{2} / 2$. Because nearly all of the mass of the plasma is in the protons, and the protons and electrons are nearly equal in number, Fermiacceleration dissipation of the turbulence into the electrons will bulk energize the electrons to a final mean energy $E_{f}$ of order $m_{p} V_{\mathrm{A}}^{2} / 2$. The observed energy spectra of impulsivephase hard X-ray bursts show that $E_{f}$ is typically of order $20 \mathrm{keV}$ (e.g., Tandberg-Hanssen \& Emslie 1988). That is, if the hard X-ray electrons are energized as we propose by dissipation of reconnection-driven MHD turbulence, then the observed hard X-ray emission requires that the Alfvén speed in the reconnection region be of a certain order of magnitude:

$$
V_{\mathrm{A}} \sim\left(2 E_{f} / m_{p}\right)^{1 / 2},
$$

from which $E_{f} \sim 20 \mathrm{keV}$ gives $V_{\mathrm{A}} \sim 2 \times 10^{8} \mathrm{~cm} \mathrm{~s}^{-1}$. This is the expected order of magnitude for the Alfven speed in the coronal body of active regions and hence in the reconnection region in flares: $V_{\mathrm{A}} \sim 2 \times 10^{8} \mathrm{~cm} \mathrm{~s}^{-1}$ for $B_{0} \sim 10^{2} \mathrm{G}$ and $n_{e} \sim 10^{10} \mathrm{~cm}^{-3}$. Thus, in this way, the expected reconnection-driven MHD turbulence is compatible with the typical hard X-ray burst observed in the flare impulsive phase.

The scale $l_{0}$ of the largest eddies of the turbulence is set by the scale of the driver of the turbulence: $l_{0}$ is of the order of the width of a reconnection-outflow jet. Each of these large eddies cascades and dissipates in about one turnover time, $\sim l_{0} / V_{\mathrm{A}}$. For dissipation by Fermi acceleration of the electrons, the turbulence cascades to the length scale at which the Fermi-acceleration electron-energization rate equals the rate at which the turbulent energy flows down the cascade. That is, the acceleration time $t_{\text {acc }}$ for the electrons in the large eddy to be bulk energized to their final energy $E_{f} \sim$ $m_{p} V_{\mathrm{A}}^{2} / 2$ by Fermi acceleration is set by the large-eddy turnover time:

$$
t_{\mathrm{acc}} \sim l_{\mathrm{o}} / V_{\mathrm{A}} .
$$

Thus, as each large eddy cascades and dissipates, its electrons are energized to a mean energy of order $m_{p} V_{\mathrm{A}}^{2} / 2$ in a burst having a duration of order $l_{0} / V_{\mathrm{A}}$.

Observations of the flare impulsive-phase radio and hard $\mathrm{X}$-ray emission with time resolution $\lesssim 0.1 \mathrm{~s}$ show that the overall burst (spanning tens to hundreds of seconds) often has strong spiky substructure on subsecond timescales (Kaufmann et al. 1980; Benz \& Aschwanden 1992;
Machado et al. 1993; Aschwanden, Schwartz, \& Alt 1995). This subsecond structure suggests that the impulsive-phase energy release is highly fragmented, occurring in many spatially distinct but temporally overlapping events (Kaufmann et al. 1980; Sturrock et al. 1984; Benz \& Aschwanden 1992; LaRosa \& Moore 1993). Each fragment releases $\sim 10^{26}$ ergs in $\sim 0.3 \mathrm{~s}$, energizing $\sim 3 \times 10^{33}$ electrons to energies $\sim 20 \mathrm{keV}$ (Benz \& Aschwanden 1992; Machado et al. 1993). Following LaRosa \& Moore (1993), we identify these observed energy release fragments with the large eddies in the reconnection-driven MHD turbulence, each $\sim 0.3 \mathrm{~s}$ hard $\mathrm{X}$-ray spike being emitted by the electrons energized in the cascade of a large eddy. For $V_{\mathrm{A}} \sim 2 \times 10^{8}$ $\mathrm{cm} \mathrm{s}^{-1}$ (required by $E_{f} \sim 20 \mathrm{keV}$ ) and $t_{\mathrm{acc}} \sim 0.3 \mathrm{~s}$, equation (3.3) gives $l_{0} \sim \frac{2}{3} \times 10^{8} \mathrm{~cm}$ for the diameter of the large eddies. For the expected electron density $n_{e} \sim 10^{10} \mathrm{~cm}^{-3}$, an eddy of this size contains $\sim 3 \times 10^{33}$ electrons. In addition, a scale of order $10^{8} \mathrm{~cm}$ is plausible for the reconnection outflows in flares (LaRosa \& Moore 1993). Thus, our expected impulsive-phase MHD turbulence is compatible with both the mean electron energy $E_{f}$ and the acceleration time $t_{\text {acc }}$ implied by the characteristic steep energy spectrum and subsecond time structure of the observed hard X-ray emission.

Now that we have in hand the spectrum, strength, and driver scale of the expected MHD turbulence, we proceed to derive the dissipation scale that these require for the turbulence to dissipate by Fermi acceleration of the electrons. For Kraichnan (or Kolmogorov) MHD turbulence (eq. [3.1]), our expression (eq. [2.7]) for the $e$-folding energization time $\tau_{E}$ for the electrons becomes

$$
\begin{gathered}
\tau_{E} \approx \frac{1}{2}\left(\frac{l_{0}}{V_{\mathrm{A}}}\right)\left[\frac{\left(2 E / m_{e}\right)^{1 / 2}}{V_{\mathrm{A}}}\right]\left(\frac{\delta l}{l_{0}}\right)^{1 / 2} \\
{\left[\tau_{E} \approx \frac{1}{2}\left(\frac{l_{0}}{V_{\mathrm{A}}}\right)\left[\frac{\left(2 E / m_{e}\right)^{1 / 2}}{V_{\mathrm{A}}}\right]\left(\frac{\delta l}{l_{0}}\right)^{1 / 3}\right],}
\end{gathered}
$$

where the electron rms speed is expressed in terms of the mean electron energy $E$. By definition of $\tau_{E}$, the rate of increase of the mean electron energy is

$$
d E / d t=E / \tau_{E} .
$$

Equations (3.4) and (3.5) together show that the rate of bulk energization $d E / d t$ increases both with decreasing $\delta l$ and with increasing $E$, whereas the $e$-folding time $\tau_{E}$ decreases with decreasing $\delta l$ but increases with increasing $E$. Integration of equation (3.5) gives

$$
t_{\mathrm{acc}}=\int_{0}^{t_{\mathrm{acc}}} d t=\int_{E_{0}}^{E_{f}}\left(\tau_{E} / E\right) d E,
$$

where $E_{0}$ is the initial mean energy of the electrons. Combining equations (3.4) and (3.6), we obtain

$$
\begin{aligned}
t_{\mathrm{acc}} & \approx\left(l_{0} / V_{\mathrm{A}}\right)\left[\left(2 / m_{e}\right)^{1 / 2} / V_{\mathrm{A}}\right]\left(\delta l / l_{0}\right)^{1 / 2}\left(E_{f}^{1 / 2}-E_{0}^{1 / 2}\right) \\
{\left[t_{\mathrm{acc}}\right.} & \left.\approx\left(l_{\mathrm{o}} / V_{\mathrm{A}}\right)\left[\left(2 / m_{e}\right)^{1 / 2} / V_{\mathrm{A}}\right]\left(\delta l / l_{0}\right)^{1 / 3}\left(E_{f}^{1 / 2}-E_{0}^{1 / 2}\right)\right] .
\end{aligned}
$$

In our case, $E_{0}$ is the mean thermal energy of the electrons in the coronal plasma entering the flare turbulence: $E_{0} \sim$ $k T_{0} \lesssim 0.3 \mathrm{keV}$ for $T_{0} \lesssim 3 \times 10^{6} \mathrm{~K}$. Hence, for $E_{f} \sim 20 \mathrm{keV}$, we have $E_{0} \ll E_{f}$, and equation (3.7) becomes

$$
\begin{aligned}
t_{\mathrm{acc}} & \approx\left(l_{0} / V_{\mathrm{A}}\right)\left[\left(2 E_{f} / m_{e}\right)^{1 / 2} / V_{\mathrm{A}}\right]\left(\delta l / l_{0}\right)^{1 / 2}=2 \tau_{E_{f}} \\
{\left[t_{\mathrm{acc}}\right.} & \left.\approx\left(l_{0} / V_{\mathrm{A}}\right)\left[\left(2 E_{f} / m_{e}\right)^{1 / 2} / V_{\mathrm{A}}\right]\left(\delta l / l_{0}\right)^{1 / 3}=2 \tau_{E_{f}}\right] .
\end{aligned}
$$




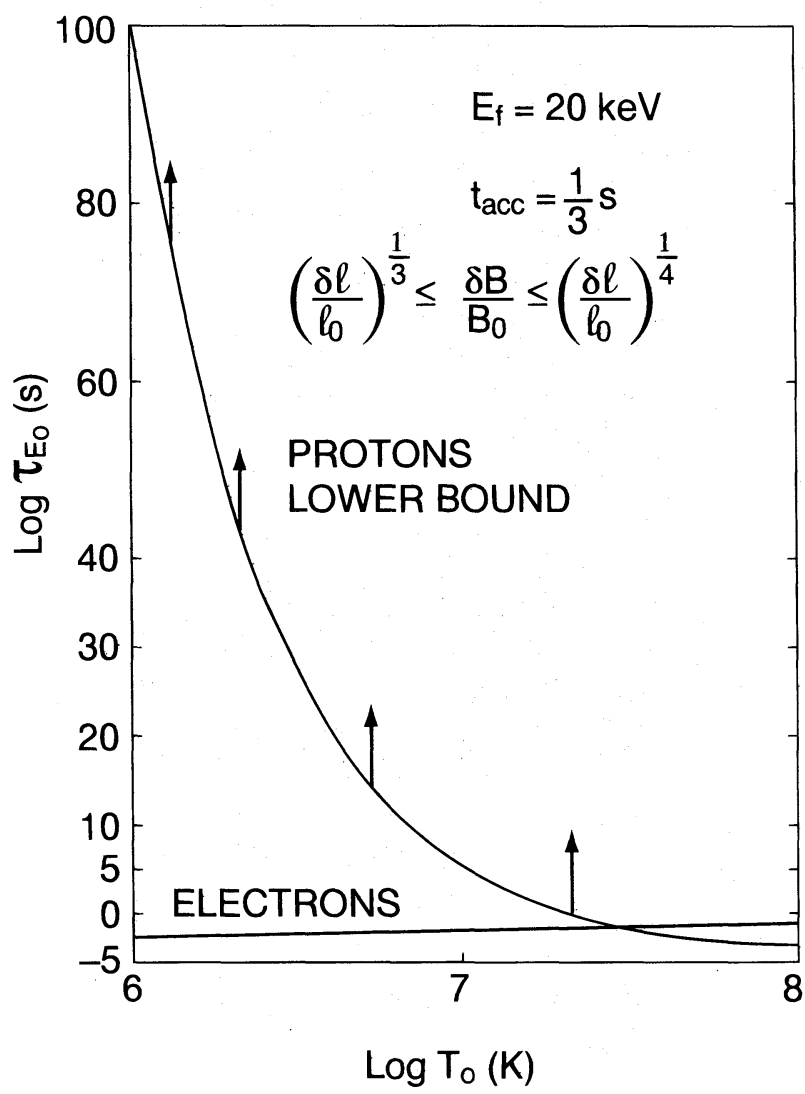

Fig. 3.-Immunity of the protons to Fermi-acceleration energization by reconnection-driven MHD turbulence in flares. The lower curve gives the electron initial $e$-folding energization time $\tau_{E_{0}}$ as a function of the initial temperature $T_{0}$ of the flare coronal plasma at the onset of the MHD turbulence. We have used MHD turbulence of the strength and scale inferred from the observed hard X-ray emission from impulsive flares $\left(E_{f}=20 \mathrm{keV}, t_{\mathrm{acc}}=\frac{1}{3} \mathrm{~s}\right)$ and that has any spectrum no steeper than Kolmogorov $\left[\delta B / B_{0}=\left(\delta l / l_{0}\right)^{1 / 3}\right]$ and no flatter than Kraichnan $\left[\delta B / B_{0}=\right.$ $\left.\left(\delta l / l_{0}\right)^{1 / 4}\right]$. The upper curve is our lower bound on $\tau_{E_{0}}$ for the protons in the same turbulence. It is seen that the protons are energized much more slowly than the electrons, certainly for any initial temperature less than about $3 \times 10^{7} \mathrm{~K}$, which is well above the expected initial temperature. Hence, there is practically no Fermi-acceleration energization of the protons as the compressive component of the cascading MHD turbulence dissipates by Fermi-acceleration bulk energization of the electrons.

That is, for initial electron mean energies much less than their final mean energy, the time for the Fermi acceleration to bulk energize the electrons to their final energy is about twice the $e$-folding time at the final energy. Finally, using equations (3.2) and (3.3) in equation (3.8), we obtain

$$
\begin{aligned}
\delta l / l_{0} & \sim m_{e} / m_{p} \\
{\left[\delta l / l_{0}\right.} & \left.\sim\left(m_{e} / m_{p}\right)^{3 / 2}\right] .
\end{aligned}
$$

Thus, for coronal plasma initially at preflare active-region temperatures in Kraichnan (Kolmogorov) MHD turbulence driven by Alfvénic flows, the dissipation scale for dissipation by Fermi acceleration of the electrons is $\sim 10^{3}\left(10^{5}\right)$ times smaller than the scale of the driving flows. Hence, in the impulsive phase of flares, where $l_{0} \sim 10^{8} \mathrm{~cm}$, the electron Fermi acceleration quenches the turbulent cascade at scales of order $10^{5} \mathrm{~cm}\left(10^{3} \mathrm{~cm}\right)$. Because this dissipation scale is at least 2 orders of magnitude larger than the ion gyroradius (for $T_{0} \lesssim 3 \times 10^{6} \mathrm{~K}$ and $B_{0} \gtrsim 10^{2} \mathrm{G}$ ), the magnetic fluctuations that do the Fermi acceleration are typically within the MHD regime of the plasma; so it is appropriate to model the flare turbulence as a purely MHD phenomenon throughout the cascade spectrum from the driver scale to the Fermi dissipation scale (LaRosa et al. 1994).

We now have an end-to-end description of plausible MHD turbulence that is capable of providing, through Fermi acceleration, the energization of the electrons for the impulsive-phase hard X-rays from flares. This Fermi acceleration is a viable mechanism for the needed electron bulk energization, provided that the turbulence is not quenched by the protons through some faster process at a scale equal to or larger than that required for the electron Fermi acceleration. In particular, there is the question of whether the Fermi acceleration can act faster on the protons than on the electrons, resulting in the protons absorbing the cascading turbulent energy first and thus blocking the energization of the electrons. We now turn to this question.

\subsection{Magnitudes of Energization Times and Their Temperature Dependence}

To assess the threat of the protons to the Fermiacceleration bulk energization of the electrons, we compare the initial $e$-folding time $\tau_{E_{0}}$ for the electrons to that for the protons. The electrons suffer no significant blockage from the protons so long as $\tau_{E_{0}}$ for the electrons is much shorter than $\tau_{E_{0}}$ for the protons.

Because of the low-beta condition of the magnetized plasma entering the flare impulsive-phase MHD turbulence, $\frac{1}{2} m_{p} V_{\mathrm{A}}^{2} \gg k T_{0}$, equation (3.8) applies and together with equation (3.4) expresses the $e$-folding time for the bulk energization of the electrons in terms of $E, E_{f}$, and $t_{\mathrm{acc}}$.

$$
\tau_{E} \approx \frac{1}{2}\left(\frac{E}{E_{f}}\right)^{1 / 2} t_{\mathrm{acc}}
$$

(This result is independent of the steepness of the turbulence spectrum because the dissipative scale for any given spectral slope adjusts to give the same $\tau_{E}$ for the given $E_{f}$ and $l_{0}$.) The electrons in the plasma entering the turbulence initially have a mean energy set by the preflare temperature $T_{0}$ of the plasma: $E_{0} \sim k T_{0}$. Thus, for $t_{\mathrm{acc}} \sim \frac{1}{3} \mathrm{~s}$ and $E_{f} \sim 20 \mathrm{keV}$, the initial $e$-folding energization time for the electrons is

$$
\tau_{E_{0}} \sim 10^{-5} T_{0}^{1 / 2} \mathrm{~s} .
$$

So $\tau_{E_{0}}$ gradually increases with increasing $T_{0}$. For $k T_{0} \ll$ $E_{f}=20 \mathrm{keV}$, we have $T_{0} \ll 2.5 \times 10^{8} \mathrm{~K}$ and $\tau_{E_{0}} \ll \frac{1}{6} \mathrm{~s}=$ $\tau_{E_{f}}=\frac{1}{2} t_{\text {acc }}$, which merely restates that the electrons are bulk energized to $20 \mathrm{keV}$ in a third of a second (we used this condition to set the value of the constant coefficient in eq. [3.11]).

For the same turbulence that provides the above electron bulk energization, our lower bound on the initial $e$-folding energization time for the protons has the following expression (from eqs. [2.20] and [3.8]):

$$
\begin{aligned}
\tau_{E_{0}} & \gtrsim \frac{1}{4}\left(\frac{m_{e}}{m_{p}}\right)^{5 / 8}\left(\frac{k T_{0}}{E_{f}}\right)^{3 / 2} \exp \left(\frac{E_{f}}{k T_{0}}\right) t_{\mathrm{acc}} \\
{\left[\tau_{E_{0}}\right.} & \left.\lesssim \frac{1}{4}\left(\frac{m_{e}}{m_{p}}\right)^{3 / 4}\left(\frac{k T_{0}}{E_{f}}\right)^{3 / 2} \exp \left(\frac{E_{f}}{k T_{0}}\right) t_{\mathrm{acc}}\right],
\end{aligned}
$$

which, with $t_{\text {acc }} \sim \frac{1}{3} \mathrm{~s}$ and $E_{f} \sim 20 \mathrm{keV}$, becomes

$$
\tau_{E_{0}} \gtrsim 10^{-16} T_{0}^{3 / 2} \exp \left(2.5 \times 10^{8} / T_{0}\right) \mathrm{s}
$$

for $T_{0} \ll 2.5 \times 10^{8} \mathrm{~K}$ and any turbulence spectrum between Kraichnan and Kolmogorov. So our lower bound on $\tau_{E_{0}}$ for 
the protons decreases very rapidly with increasing initial temperature for $T_{0} \ll 2.5 \times 10^{8} \mathrm{~K}$. However, at these temperatures, the value of this lower bound stays well above the value of $\tau_{E_{0}}$ for the electrons. For example, for $T_{0}=10^{7} \mathrm{~K}$, equation (3.13) bounds the proton $\tau_{E_{0}}$ above $\sim 5 \times 10^{5} \mathrm{~s}$.

Figure 3 shows the electron $\tau_{E_{0}}$ (eq. [3.11]) and our lower bound on the proton $\tau_{E_{0}}$ (eq. [3.13]) plotted against the initial temperature. These curves demonstrate the main point of this paper: for any reasonable preflare temperature $\left(T_{0} \lesssim 10^{7} \mathrm{~K}\right)$, Fermi-acceleration energization of the protons is much too slow to appreciably diminish the Fermi-acceleration energization of the electrons.

\section{DISCUSSIONS AND CONCLUSIONS}

In this paper we have shown that for the expected magnetic field strength, plasma density, and initial temperature of the plasma entering the reconnection-driven MHD turbulence in flares, electron Fermi acceleration by large-scale magnetosonic waves $\left(\delta l \lesssim 10^{3} \mathrm{~cm}\right)$ is many orders of magnitude more efficient than proton Fermi acceleration. At temperatures below $\sim 10^{8} \mathrm{~K}$, the proton thermal speed is less than the Alfvén speed. For particle speeds below the Alfvén speed, the particle pitch-angle distribution in the frame of the accelerating MHD waves is so collapsed in the direction of the magnetic field that mirroring is negligible and the particles cannot exchange energy with the waves. We therefore conclude that if MHD turbulence is produced by the flare energy release process, then the compressive component of this turbulence is channeled into the electrons via Fermi acceleration because of the higher thermal velocity of the electrons.

We emphasize that since electron Fermi acceleration occurs at physical scales $\gtrsim 10^{3} \mathrm{~cm}$, other dissipation mechanisms will not be important and it is the Fermi mechanism which terminates the compressive component of the cascade. It is often assumed that dissipation becomes important only when fine-scale gradients in velocity or (in the MHD case) when fine-scale currents develop. Gradients in velocity result in viscous dissipation in a turbulent hydrodynamic flow, and a combination of resistive and viscous dissipation can occur in a turbulent MHD flow (e.g., Biskamp \& Welter 1989). However, the particles also interact with large-scale turbulent magnetic fluctuations through magnetic mirroring. In fact, mirroring is stronger for larger scale, larger amplitude fluctuations, since the loss-cone angle scales as the square root of magnetic field fluctuation relative to the background magnetic field. We find that Fermi acceleration dissipation occurs on physical scales $\gtrsim 10^{3} \mathrm{~cm}$. Resistive and viscous dissipation of the cascading energy could occur only on scales significantly smaller, since the current density and velocity gradients on this scale are too weak. The current density $j$ can be estimated by $j \sim \delta B / \delta l$. For $\delta B \sim 10^{-1 / 3} \mathrm{G}$ and a length scale $\delta l \sim 10^{3}$ $\mathrm{cm}$, resistive current dissipation only dissipates $\sim 10^{21}$ ergs in a large-scale turbulent eddy (volume $\sim 10^{24} \mathrm{~cm}^{3}$ ) in our flare turbulence. The Fermi process results in a dissipation of $\sim 10^{26}$ ergs in such an eddy. Even if the resistivity is anomalously enhanced by several orders of magnitude, resistive dissipation is still negligible compared to Fermi acceleration. Similar considerations show that at scales of $\sim 10^{3} \mathrm{~cm}$ in our MHD turbulence, viscous dissipation is also much weaker than the Fermi process. Hence, under coronal flare conditions turbulent MHD wave energy dissipates into electron energy by mirroring the electrons rather than dissipating via resistivity or viscosity. This is a major difference between laboratory plasmas and the reconnection-driven turbulence in solar flares. In the laboratory large-scale flows and modes are suppressed to maintain global stability. Microinstabilities generate plasma turbulence (as opposed to MHD turbulence) on small scales, of the order of the particle gyroradii. Consequently, resistive dissipation is important in the laboratory. However, in the solar flare reconnection-driven outflow it is the large-scale MHD modes that are excited, and it is not clear that small-scale plasma turbulence (strong enough for significant dissipation) is generated except in the very limited neighborhoods of the actual reconnection. Thus, in the solar flare case, it appears that resistive current dissipation is not important for the electron bulk energization.

Our scenario for bulk energization of electrons by the Fermi process rests on two crucial assumptions: the first is that the flare energy release process generates large-scale MHD turbulence that cascades to smaller scales. The second assumption is that there is sufficient pitch-angle scattering to maintain an isotropic pitch-angle distribution throughout the acceleration process. These assumptions are now critically discussed.

\subsection{Generation of MHD Turbulence and the Importance of a Cascade}

The conditions for a magnetic Kelvin-Helmholtz or shear-flow instability have been studied by numerous investigators (e.g., Chandrasekhar 1961; Miura \& Pritchett 1982; Roy Choudhury \& Lovelace (1986). When the magnetic field is parallel to the shear flow, it has a stabilizing effect, since the flow must do work bending the field. According to the studies cited above, shear flows parallel to a magnetic field become unstable when the velocity difference across the layer is $\geq 2 V_{\mathrm{A}}$. Roberts, Goldstein, \& Ghosh (1992) have recently shown through numerical simulation the development of a turbulent cascade for this condition for two-dimensional incompressible MHD. Chiueh \& Zweibel (1987) have analytically investigated the specific case of the shear stability of a reconnection outflow. Their study included a solution for the magnetic field profile across the width of the jet. The magnetic field in the interior of a reconnecting jet can be considerably weaker than the surrounding field. Since the jet velocity is determined by the Alfvén speed of the reconnecting field, not the interior field, their expectation was that a reconnection jet should be shear-flow unstable. Their analysis supported this insight, and they found instability for flow speeds that exceeded the local (interior) Alfvén speed. There has also been some additional theoretical and numerical study on the closely related problem of the stability of jets associated with surges (Carbone, Einaudi, \& Veltri 1987; Dahlburg \& Karpen 1994). The surge is modeled as a jet flowing through a sheared magnetic field. Carbone et al. (1987) originally proposed that a transition to turbulence could account for the observed rapid deceleration of surges. Recently, these flows have been shown to undergo a transition to turbulence through a secondary three-dimensional instability on a two-dimensional saturated state (Dahlburg \& Karpen 1994). Collectively these results support our contention that flare reconnection outflows in the solar corona are unstable and MHD turbulent.

Once an instability is excited, it is crucial that a cascade of turbulent energy to smaller scales also occurs. Some 
recent work on Fermi acceleration of electrons by largescale MHD turbulence by de La Beaujardière \& Zweibel (1989) and Zweibel \& de La Beaujardière (1990) indicated that Fermi acceleration could not account for electron energization. Their work differed from previous work in two respects: first they assumed that the accelerating turbulence consisted of the large-scale wave modes of a cylindrical coronal loop. Second, instead of using quasi-linear theory, they calculated the acceleration rate by directly integrating the electron orbits using the guiding center approximation. They found very little energization even for timescales long compared to observed acceleration times (Zweibel \& de La Beaujardière 1990). This result is at variance with our work. The discrepancy may be due to the fact that Zweibel \& de La Beaujardière (1990) did not consider a cascade of the MHD wave energy to shorter wavelengths. The acceleration time increases directly with the wavelength of the accelerating waves; the longer the wave, the more time required to traverse the wave. Hence, long-wavelength loop waves are very inefficient accelerators. As outlined in $\S 3$, in a turbulent cascade the energy is transferred to successively smaller scales until some dissipation mechanism is initiated. As wave energy cascades to smaller scales, the acceleration time due to the Fermi process approaches the eddy turnover time. It is when these times are equivalent that the cascade terminates. We showed that at scales of $10^{5}-10^{3} \mathrm{~cm}$ the acceleration time matches the eddy turnover time. We therefore conclude that, given the turbulent cascade expected in flares, the Fermi process can bulk energize the electrons on the observed timescales.

\subsection{Pitch-Angle Scattering}

Beyond the question of the generation of turbulence, the most significant question challenging electron energization by the Fermi process is pitch-angle scattering. The Fermi mechanism boosts only the parallel momentum and thus collapses the pitch-angle distribution of the accelerated particles. In order that the particles continue to mirror and gain energy, the Fermi process requires efficient pitch-angle scattering to repopulate the large pitch angles (e.g., Melrose 1974; Achterberg 1981). Melrose (1974) pointed out that resonant wave-particle scattering was the only mechanism fast enough to maintain an isotropic pitch-angle distribution on observed acceleration timescales. (Coulomb collisions for particles above about $1 \mathrm{keV}$ are too slow to scatter particles on the observed timescales.) Thus, one must either invoke an external source of small-scale plasma waves or assume that the velocity anisotropy that develops as a result of the acceleration process leads to plasma instability and self-generated waves. Possibilities for external small-scale plasma turbulence include plasma instability associated with the reconnection process or generation by nonlinear wave-wave interactions in a cascade. One difficulty with generating the scattering waves in the reconnection is that the volume of the reconnection region is small compared to the volume of the reconnection outflows, and the scattering waves must be present throughout the turbulent outflow. The second possibility implies that wave energy cascades from the largest scales in the flare $\left(\sim 10^{8} \mathrm{~cm}\right)$ to the electron gyroradius $(\sim 50 \mathrm{~cm})$. This process, however, has not been investigated.

At this point it appears that the most realistic origin for the scattering waves is self-generation through a velocity anisotropy instability. An electron distribution with a parallel anisotropy is observed in the laboratory to destabilize through the anomalous Doppler resonance $(\omega+\Omega=$ $k_{\|} V_{\|}$), resulting in the generation of electrostatic lowe hybrid waves (Liu \& Mok 1977; Moghaddam-Taaheri et al. 1985). The lower hybrid waves will in turn pitch-anglescatter the electrons and remove the anisotropy. The wave growth is therefore limited by the back-reaction of the waves on the particle velocity distribution. We expect that this system evolves to a state of marginal stability in which all three processes (Fermi acceleration, wave growth, and pitch-angle scattering) operate simultaneously in equilibrium. In this case the wave scattering will not be so strong as to prevent the Fermi acceleration. The details of this process need to be investigated self-consistently and will be the subject of future work.

Although we do not yet rule out the possibility of externally generated waves providing scattering, self-generated wave scattering offers the possibility for a self-sufficient acceleration model. According to Melrose (1974), the resonance condition for electrons interacting with parallelpropagating transverse electromagnetic waves require the electron velocity to exceed $\left(m_{p} / m_{e}\right)^{1 / 2} V_{\mathrm{A}}$, which corresponds to an energy exceeding $25 \mathrm{keV}$ for coronal conditions. Melrose (1974) therefore concluded that the Fermi process was only important as a second-stage acceleration mechanism and could not be responsible for electron energization to $\sim 20 \mathrm{keV}$ from $\sim 0.1 \mathrm{keV}$. However, recent work by Steinacker \& Miller (1992), Miller \& Steinacker (1992), and Hamilton \& Petrosian (1992) finds a much lower threshold condition for resonance. These authors reconsidered the resonance condition for electrons with parallel-propagating transverse electromagnetic waves (i.e., right- and leftcircularly polarized waves, such as whistler waves). The full resonance condition is

$$
\omega-k v_{z}=-s \epsilon \Omega_{0} / \gamma,
$$

where $\omega$ is the wave-frequency, $\Omega$ is the cyclotron frequency, $\epsilon$ is the particle charge, $s=+1$ or -1 for right-hand or left-hand circular polarization, and $\gamma$ is the Lorentz factor. Earlier treatments neglected $\omega$ in relation to $k v_{z}$ and $\Omega_{0} / \gamma$. Steinacker \& Miller (1992) showed explicitly when this approximation breaks down and concluded that for coronal conditions the full resonance condition must be used. Even when thermal damping of the waves is included, Miller \& Steinacker (1992) find that electrons of energy $\sim 10 \mathrm{kT}$, which corresponds to $1 \mathrm{keV}$ in the corona, can resonate with whistler and R-waves close to the gyrofrequency. This result is significantly below the previously accepted value based on the condition $V \geq\left(m_{p} / m_{e}\right)^{1 / 2} V_{A}$ and suggests that low-energy electrons could resonate and therefore generate scattering waves. If so, the Fermi process could accelerate particles from the thermal background, since below $1 \mathrm{keV}$, Coulomb collisions are fast enough to maintain isotropy.

\subsection{Conclusion}

In this work we have shown that for the expected MHD turbulence in a flare reconnection outflow electron Fermi acceleration is many orders of magnitude faster than proton Fermi acceleration. This means that if such large-scale strong MHD turbulence is generated and cascades, the Fermi mechanism will dissipate the compressive component only into the electrons and not into the protons. We also found that the electron Fermi acceleration is strong enough 
to be the process that terminates the compressive component of the cascade. We conclude that Fermi acceleration is a promising electron bulk energization mechanism for solar flares. It can account for the efficiency, the timing, and the global energetics of the flare electron energization as inferred from hard X-ray observations.

An additional test of the Fermi energization model will be to calculate the electron energy spectrum resulting from Fermi acceleration. There is some indirect evidence that the Fermi mechanism can account for observed spectra. In our scenario of reconnection-driven MHD turbulence the wave amplitudes are in the nonlinear regime, so a test-particle approach will be required to compute the spectrum. However, Miller, LaRosa, \& Moore (1995) have recently calculated, using quasi-linear theory, the electron energy spectrum resulting from small-amplitude Fermi acceleration in a turbulent MHD cascade. In the small-amplitude (quasi-linear) case, the turbulent energy density is several orders of magnitude smaller than in the large-amplitude case. To match the flare hard X-ray observations, the small amplitude model requires a low level of turbulence to be excited over a much larger volume $\left(\sim 10^{27} \mathrm{~cm}^{3}\right)$ than one of our large-scale eddies $\left(\sim 10^{24} \mathrm{~cm}\right)$. The resulting energy spectra were found to be hard enough to be compatible with the observed spectra, a major obstacle for many models. A greater turbulent energy density should not alter this finding. Hence this result for the small-amplitude case is promising for our large-amplitude scenario.

Collectively all of these results are encouraging. The most important issue that remains to be investigated is the pitchangle scattering of the accelerated electrons which is required for Fermi acceleration to occur. Pitch-angle scattering by self-generated waves will be the considered in future work.

We thank Spiro Antiochos for initially pointing out the potential threat to the electron bulk energization by proton Fermi acceleration. T. N. L. was supported by a NASA/ JOVE grant to Kennesaw State College, R. L. M. was supported by NASA's Office of Space Science through the Solar Physics Branch of its Space Physics Division. J. A. M. was supported by the NSF Solar Terrestrial Physics Program and the NASA Cosmic and Heliospheric Physics Program.
Achterberg, A. 1981, A\&A, 97, 259

Aschwanden, M. J., Schwartz, R. A., \& Alt, D. A. 1995, ApJ ;47, 923

Benz, A. O.; \& Aschwanden, M. J. 1992, in Eruptive Solar Flares, ed. Z. Svestka, B. V. Jackson, \& M. E. Machado (Berlin: Springer), 106

Biskamp, D., \& Welter, H. 1989, Phys. Fluid B; 1, 1964

Carbone, V., Einaudi, G., \& Veltri, P. 1987, Sol. Phys., 11, 31

Chandrasekhar, S. 1961, Hydrodynamic and Hydromagnetic Stability (Oxford: Clarendon)

Chiueh, T., \& Zweibel, E. G. 1987, ApJ, 317, 900

Dahlburg, R. B., \& Karpen, J. T. 1994, ApJ, 434, 766

de La Beaujardière, J. \& Zweibel; E. G. 1989, ApJ, 336, 1059

Emslie, A. G., \& Brown, J. C. 1985, ApJ, 295, 648

Hamilton, R. J., \& Petrosian, V. 1992, ApJ, 398, 350

Kaufmann, P., Strauss, F. M., Opher, R., \& Laporte, C. 1980, A\&A, 87, 58

Kraichnan, R. H. 1965, Phys. Fluids, 8, 1385

LaRosa, T. N., \& Moore, R. L. 1993, ApJ, 418, 912

LaRosa, T. N., Moore, R. L., \& Shore, S. N. 1994, ApJ, 425, 856

Liu, C. S., \& Mok, Y. 1977, Phys. Rev. Lett., 38, 162

Machado, M. J., Ong, K. K., Emslie, A. G., Fishman, G. J., Meegan, C., Wilson, R., \& Paciesas, W. S. 1993, Adv. Space Res., 13(9), 175

Martres, M. J., \& Bruzek, A. 1977, in Illustrated Glossary for SolarTerrestrial Physics, ed. A. Bruzek \& C. J. Durrant (Dordrecht: Reidel), 53

Melrose, D. B. 1974, Sol. Phys., 37, 353

1986, Instabilities in Space and Laboratory Plasmas (Cambridge: Cambridge Univ. Press)

. 1992, in Eruptive Solar Flares, ed. S. Svestka, B. V. Jackson, \& M. E. Machado (Berlin: Springer), 147

Miller, J. A., LaRosa, T. L., \& Moore, R. L. 1996, ApJ, 460, 445

Miller, J. A., \& Roberts, D. A. 1996, ApJ, in press

Miller, J. A., \& Steinacker, J. 1992, ApJ, 399, 284

Miura, A., \& Pritchett, P. L. 1982, J. Geophys. Res., 87, 7431

\section{REFERENCES}

Moghaddam-Taaheri, E., Vlahos, L., Rowland, H. L., \& Papadopoulos, K. 1985, Phys. Fluids, 28, 3356

Moore, R. L. 1992, in The Astronomy and Astrophysics Encyclopedia, ed.

S. P. Maran (New York: Van Nostrand Reinhold), 637

Moore, R. L., \& Fung, P. C. W. 1972, Sol. Phys., 23, 7

Moore, R. L., Hurford, G. J., Jones, H. P., \& Kane, S. R. 1984, ApJ, 276 379

Moore, R. L., LaRosa, T. N., \& Orwig, L. E. 1995, ApJ, 438, 985

Moore, R. L., \& Roumeliotis, G. 1992, in Eruptive Solar Flares, ed. Z. Svestka, B. V. Jackson, \& M. E. Machado (Berlin: Springer), 69

Priest, E. R., \& Forbes, T. E. 1992, J. Geophys. Res., 97, 16757

Ramaty, R., et al. 1980, in Solar Flares: A Monograph from Skylab Solar Workshop II, ed. P. A. Sturrock (Boulder: Colorado Assoc. Univ. Press) 117

Roberts, D. A., Goldstein, M. L., \& Ghosh, S. 1992, J. Geophys. Res., 97, 17115

Rosner, R., et al. 1991, in Working Papers, Astronomy and Astrophysics

Panel Reports (Washington DC: National Academy Press), chap. 9

Roy Choudhury, S., \& Lovelace, R. V. E. 1986, ApJ, 302, 188

Spitzer, L. 1962, Physics of Fully Ionized Plasmas (New York: Wiley)

Steinacker, J., \& Miller, J. A. 1992, ApJ, 393, 764

Sturrock, P. A., ed. 1980, Solar Flares: A Monograph from Skylab Solar Workshop II (Boulder: Colorado Assoc. Univ. Press)

Sturrock, P. A., Kaufmann, P., Moore, R. L., \& Smith, D. F. 1984, Sol. Phys., 94, 341

Sweet, P. A. 1969, ARA\&A, 7, 149

Tandberg-Hanssen, E., \& Emslie, A. G. 1988, The Physics of Solar Flares (Cambridge: Cambridge Univ. Press)

Vaiana, G. S., \& Rosner, R. 1978 ARA\&A, 16, 393

Verma, M. K. 1994, Ph.D. thesis, Univ. of Maryland

Wentzel, D. G. 1963, ApJ, 137, 135

Zweibel, E. G., \& de La Beaujardière, J. 1990, Geophys. Res. Lett., 17, 2051 